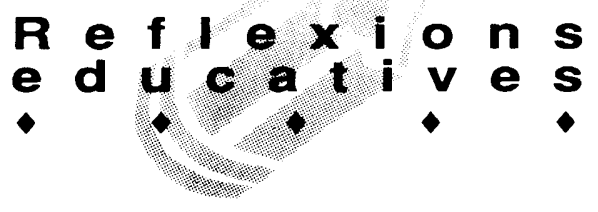

\title{
ELS CIENTÍFICS A L'ESCOLA PRIMÀRIA
}

\author{
Agustí Salvat Altés. Àrea de Didàctica de les Ciències Experimentals \\ José Sánchez Real. Escola de Magisteri. València
}

Els nens, ja des de petits, estan acostumats a sentir parlar de savis i cientifics (reals o ficticis) i a veure les seves imatges a les pel.lícules i als còmics. A més a més, a mesura que s'endinsen en l'escolarització obligatòria, els textos de ciències els presenten algunes de les biografies de les figures científiques més rellevants. Per altra part, conèixer alguns dels personatges científics més importants, així com les aportacions tecnològiques valuoses, és unvalor cultural que s' ha de seguir potenciant. Davant aquests fets cal reflexionar sobre algunes qüestions: ¿recorden, passat un cert temps, els noms dels científics estudiats? ¿associen el nom amb el personatge? ¿relacionen el nom amb la contribució més important del científic? ¿recorden algun fet caracterísitic del científic?. Per altra part, atesa l'enorme imaginació i fantasia dels nens, ¿són capaços d'inventar noms de científics? ¿qualifiquen com a científics personatges que tenen poca relació amb la ciència i la tècnica?

\section{Antecedents}

No hi ha dubte que el saber científic s'ha convertit en model del coneixement del nostre temps $i$ alguns conceptes s'han tornat actualment indispensables per comprendre i poder opinar sobre alguns dels temes sotmesos a debat permanent: l'energia en totes les seves formes possibles, el medi ambient, la investigació de l'espai, la cosmologia, la revolució informàtica il'origen de la vida, entre altres. En definitiva, sembla que la ciència juga un paper cultural que encara no es veu amb suficient claredat per la majoria de la gent - potser perquè el tema se li escapa-però si per algunes institucions públiques. Aixi, la Generalitat de Catalunya, preveient aquesta necessitat, creà al seu dia una comissió amb l'objectiu de potenciar la cultura científica a Catalunya'. D'entre el conjunt de preguntes utilitzades per detectar l'cíndex de cultura científica" del públic, n'hi havia una sobre el coneixement o record del nom de científics d'àmbit mundial. Els resultats obtinguts es donaren a conèixer al diari "La Vanguardia" i val a dir que només la meitat $(53,4 \%)$ dels enquestats recordaven algun nom, mentre que la resta no en sabien cap. La llista l'encapçalava Albert Einstein $(25,3 \%)$, seguit per Fleming $(10,4 \%)$, Newton $(6,6 \%)$, Severo Ochoa (5,4\%), Pierre i Marie Curie (4,6\%), Pasteur
$(3,4 \%)$, Edison $(3,1 \%)$, Hawking (2,9\%), Ramón y Cajal $(2,1 \%)$ i Joan Oró $(2,0 \%)$.

L'any 1991, a Oviedo, durant els XII Encuentros de Didáctica de las Ciencias Experimentales, es presentà una comunicació ${ }^{2}$ en la qual s'informava dels resultats obtinguts amb 140 alumnes de 3r., 4rt. i 5è. curs de la llicenciatura en Química, al demanar-los que escrivissin el nom de personatges històrics que haguessin desenvolupat el seu treball en el camp científic o tècnic. La conclusió a què arribaren els autors fou que els tres científics més citats coincideixen amb els de "La Vanguardia". És a dir, Einstein, Newton i Marie Curie, en aquest ordre.

\section{Sondeig entre els nens}

Per detectar quins eren els científics que els nens recordaven, durant el passat curs (1991/92) es realitzà una exploració entre els alumnes dels cursos superiors de cinc centres d'E.G.B. de Tarragona i Reus. En total intervingueren 872 escolars, desglossats, per cursos, en proporcions semblants: 161 de 5 è., 233 de $6 \dot{\text {., }} 251$ de 7 è. i 227 de 8è.

La prova consistí a contestar, per escrit i durant cinc minuts, la pregunta: Digues el nom de personatges que hagin fet algun descobriment científic útil per a la humanitat. També s'indicà als alumnes que podien anotar

\section{P.}

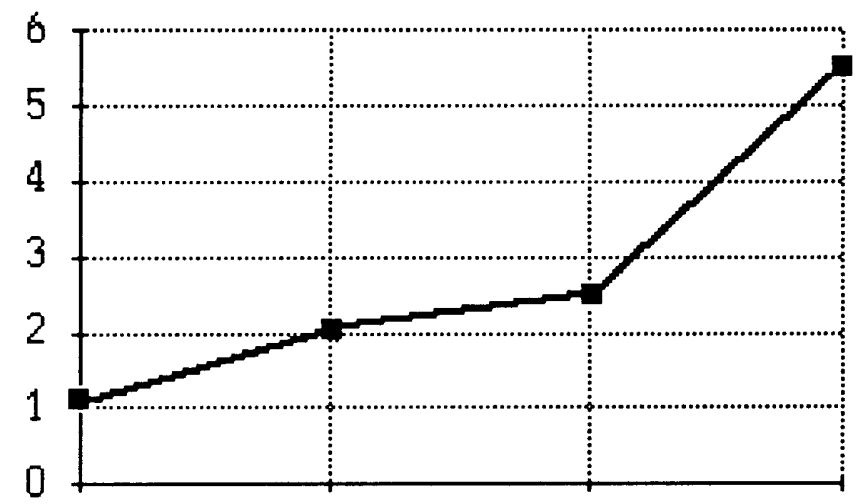

5è. EGB 6è̀. EGB 7è. EGB 8è. EGB

Gràfic 1. Index de productivitat per cursos $(\mathrm{N}=872)$ 


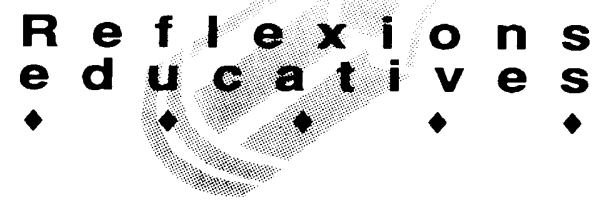

què era allò que havia descobert el personatge proposat. Amb la finalitat d'obtenir dades espontànies i fiables, s'avisà que la prova era de caràcter anònim i no tindria cap mena d'incidència en les qualificacions escolars.

\section{Resultats obtinguts}

La proporció de nens que donaren un o més noms assoli el $84,1 \%$, mentre que la resta, el $15,9 \%$, deixaren la qüestió en blanc.

Com a mitjana, el nombre de científics citats per cada nen es mostra al gràfic 1 , on "l.P." representa l'«index de productivitat", que s'ha calculat dividint el nombre total de cientifics citats entre el nombre d'alumnes que han contestat l'enquesta. La forma creixent del gràfic es totalment lògica, ja que el record creix amb l'edat: des de 1,5 per als alumnes de 5è. fins al 5,5 dels de $8 \dot{e}$., amb un salt important entre $7 \dot{e}$. i 8 è., perquè es passa de 2,5 a 5,5. Globalment s'ha obtingut una productivitat de tres noms per alumne.

Al gràfic 2 es mostra la productivitat per centres $i$ nivells. Cal advertir que, per diversos inconvenients, l'enquesta no es passà als alumnes de 5è. d'E.G.B. de l'escola $\mathrm{C}$.

A la taula 1 es donen, en ordre decreixent de vegades amb què són citats, els noms dels científics amb un percentatge superior a l'u per cent. El resultat obtingut és, en certa manera, sorprenent, ja que el científic que ocupa el primer lloc de la classificació (Albert Einstein) no acostuma a sortir als textos escolars. Una de les raons podria ser que la teoria de la relativitat no és objecte d'estudi a l'ensenyament elemental. En segona posició hi ha el físic i inventor nord-americà (Thomas Alva Edison) que ideà la làmpada d'incandescència i construí el primer tren elèctric. El segueix el britànic Isaac Newton, pare de la física clàssica, l'estudi de la qual s'inicia a l'últim curs d'E.G.B.

La resta de científics indicats pels nens es llisten a la taula 2 en ordre decreixent de freqüència amb què es mencionen.

\begin{tabular}{|c|c|}
\hline Nom & $\%$ \\
\hline 1. Einstein, A. & 11,01 \\
\hline 2. Edison, T.A. & 8,03 \\
\hline 3. Newton, I. & 5,49 \\
\hline 4. Gutenberg, J.G. & 4,86 \\
\hline 5. Bell, A.G. & 4,04 \\
\hline 6. Fleming, $A$. & 3,49 \\
\hline 7. Arquimides & 3,13 \\
\hline 8. Galilei, Galileo & 2,98 \\
\hline 9. Pasteur, L. & 2,86 \\
\hline 10. Darwin, C.R. & 2,78 \\
\hline 11. Marconi, G. & 2,47 \\
\hline 12. Watt, J. & 2,39 \\
\hline 13. Morse, S.F.B. & 2,35 \\
\hline 14. Diesel, $R$. & 1,88 \\
\hline 15. Nobel, A. & 1,80 \\
\hline 16. Stevens, R. & 1,65 \\
\hline 17. Lumière, $A$. & 1,53 \\
\hline 18. Lucreci & 1,37 \\
\hline 19. Volta, A & 1,18 \\
\hline 20. Mendeleiev, D. & 1,02 \\
\hline 21. Pascal, B. & 1,02 \\
\hline
\end{tabular}

Taula 1. Nombre de cops que és citat un determinat científic, en percentatge $(\%)$ $i$ en freqüència absoluta (f).

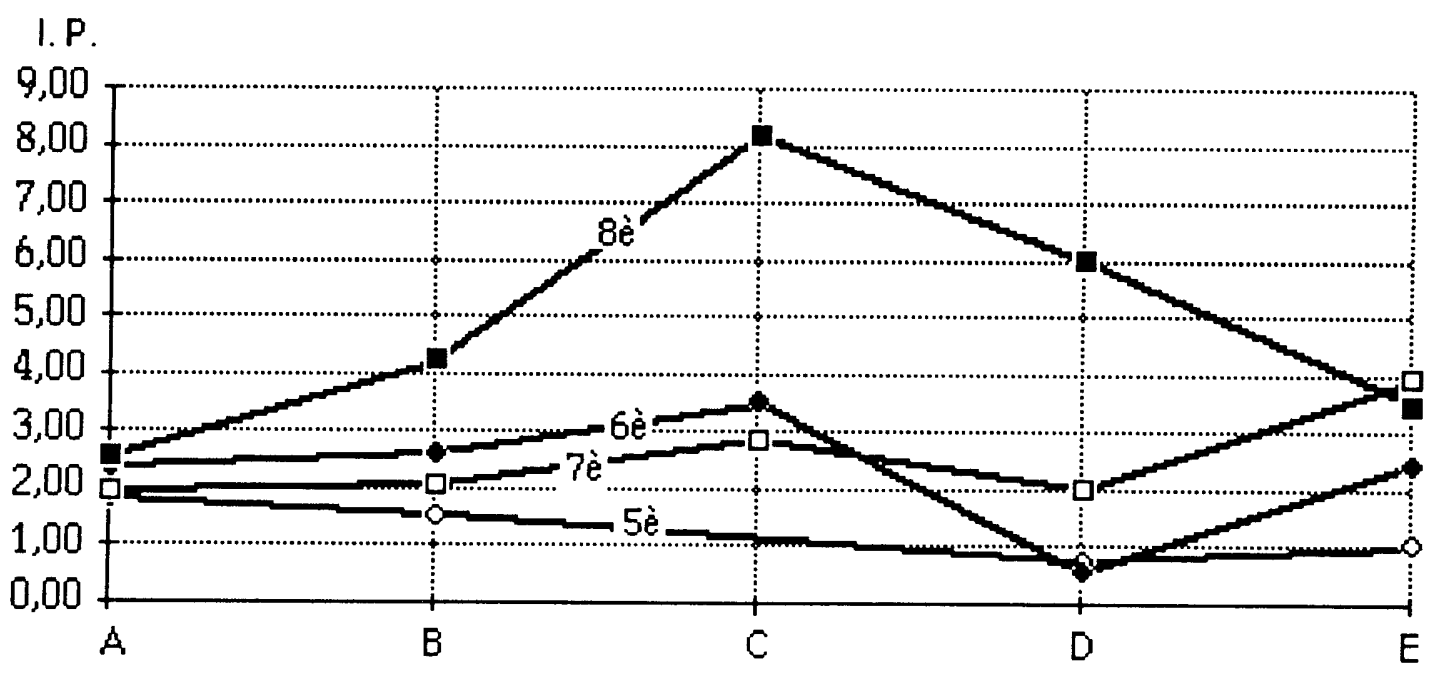

Gràfic 2. Index de productivitat per centres $(A, B, C, D$ i E) i per cursos 


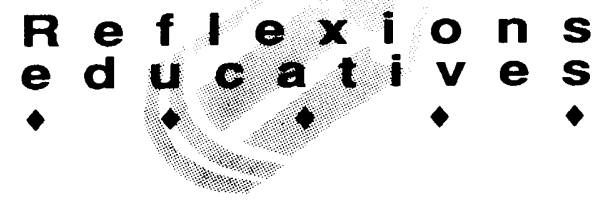

Sembla que els joves alumnes no han fet gaire cas al que se'ls demanava, perquè una tercera part dels científics de la taula 1 no han fet cap mena d'aportació científica que hagin tingut una utilitat immediata per a la humanitat. A la taula 2 la situació encara s'accentua més, ja que la proporció assoleix, si fa o no fa, la meitat dels casos. En comptades ocasions la resposta és força sorprenent, ja que hom cita Lucreci (divuitena posició), poeta llatí que deixà incomplet el poema filosófico-didàctic De rerum natura on s'exposava l'origen dels cossos, les ànimesi el món natural. Elfet que s'anomenin personatges

22. Ramón y Cajal, S.
23. Dalton, J.
24. Servet, M.
25. Ohm, G.S.
26. Torricelli, E.
27. Monturiol, N.
28. Faraday, M.
29. Curie, Marie
30. Peral, I.
31. Siemens, E.W.
32. Kepler, J.
33. Aristotil
34. da Vinci, L.
35. Bacon, R.
36. Cierva, Juan de la
37. Copernic, N.
38. Joule, J.P.
40. Halley, E.
41. Hawking, S.
42. Hooke, R.

\author{
63. Colom, C. \\ 64. Pitàgores \\ 65. Armstrong, $\mathrm{N}$. \\ 66. Mozart, W.A. \\ 67. Tales de Milet \\ 68. Verne, J. \\ 69. Home primitiu, 1 \\ 70. Lindberg, $C$. \\ 71. Elcano, J.S. \\ 72. Frankenstein \\ 73. Gandhi, M.K. \\ 74. Presley, E. \\ 75. Beethoven \\ 76. Gorbatxov, M. \\ 77. Marx, K. \\ 78. Picasso, $P$. \\ 79. Plató \\ 80. Polo, Marco \\ 81. Gaudi, A. \\ 82. Proust, $M$. \\ 83. Socrates \\ 84. Bach, J.S.
}

\author{
85. Bogart, $H$. \\ 86. Cela, C.J. \\ 87. Cervantes. M. de \\ 88. Dali, S. \\ 89. Disney, W. \\ 90. Fenicis, els \\ 91. Guillotin, J.I. \\ 92. Hitler, A. \\ 93. Lenin \\ 94. Lewis, Carl \\ 95. Lincoln, A. \\ 96. Marx, germans \\ 97. Miquel Angel \\ 98. Popeye \\ 99. Rodríguez de la F. \\ 100. Rotterdam, E. de \\ 101. Shakespeare, $W$. \\ 102. Sumeris, els \\ 103. Ulisses \\ 104. Vespuci, A. \\ 105. Wells, H.G.
}

Taula 3. Llistat de personatges d'altres camps
que s'han catalogat com a científics.

Vanguardia" ocupava la desena posició, no és anomenat per cap dels estudiants enquestats.

\section{¿"Clentifics" especials?}

El cas de Stephen Hawking i Isaac Asimov es mereix un comentari a banda. És possible que el primer d'ells, Hawking, siguiconegut per la població escolar més per la imatge que els mitjans de comunicació han mostrat del seu deplorable estat físic que pel seu llibre: Historia del tiempo, d'un contingut inintel.ligible pels nens. Un alumne de 6è. d'E.G.B. escriu al seu full de qüestionari: "Stephen Walcking (pretenia escriure Hawking), que té una teoria de com es va fer l'espai, que és molt bona. Té molt mèrit perquè és un minusvàlid i no pot parlar, però gràcies a la seva cadira de rodes pot parlar i moure's pertot arreu". Aquest comentari, com ja s'havia insinuat al començament, és un reflex de l'impacte que tenen els mitjans de comunicació social i l'entorn extrascolar sobre els coneixements que el nen ja aporta a l'escola.

Pel que fa a Asimov, malgrat que a les respostes dels alumnes no es cita cap de les seves contribucions científiques, és possible que es conegui més per la seva prolífica labor com a escriptor de ciència ficció i de divulgació científica que per les escasses aportacions 


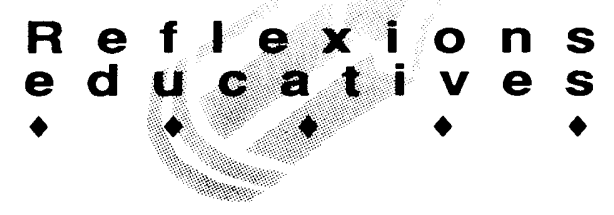

originals que ha fet al coneixement científic.

\section{Científics inventats}

Molt sovint (un $14 \%$ de les vegades) es dóna el nom d'un personatge (real o imaginari) que no correspon al de cap científic. A la taula 3 es relacionen tots els d'aquest tipus que han sorgit durant el sondeig, posats en ordre decreixent de freqüència absoluta.

Cristòfor Colom encapçala la classificació, mencionat 177 cops ( $7 \%)$, percentatge que és superior al de científics com el mateix Newton. A la llista també hi ha personatges i pobles de l'antiguitat (Pitàgores, Plató, Sòcrates, i Tales de Milet), personatges de ficció (Popeye i Frankenstein), pobles de l'antiguitat (l'home primitu, els fenicis i els sumeris), actors de cine (Humprey Bogart i els germans Marx), productors de cine (Walt Disney), pintors (Miquel Àngel, Picasso i Dalí), músics (Mozart, Bach, Beethoven i Elvis Presley), esportistes (Lewis, C.), escriptors (Cervantes, Shakespeare, Proust i C. J. Cela), filòsofs (Marx), humanistes (Erasm de Rotterdam), polítics de signe molt divers (Hitler, Lenin, Gandhi, Lincoln i Gorbatxov), arquitectes (Gaudi), naturalistes (Rodríguez de la Fuente), viatgers (Marco Polo), navegants (J. S. Elcano), aviadors (Lindberg) i astronautes (Armstrong).

És evident que en tot procés d'aprenentatge es produeixen desviacions i imprecisions que fan que l'après no es correspongui amb el disitjat. Amb els noms dels científics succeeix quelcom semblant. El nen, en el procés de memorització de noms, invents, descobriments ivincles entre ells, fa la sensació que no acaba de fixar, exactament, les connexions entre tots ells, i quan reinverteix el procediment per extreure les dades emmagatzemades al cervell, provoca un seguit de confusions que poden arribar a ser força sorprenents.

\section{Confusions}

En primer lloc cal mencionar les confusions produïdes a l'escriptura dels noms. Alguns d'ells tenen una grafia realment complexa i no ha d'estranyar que s'escrigui "Wotenburg" (per Gutenberg) i "Haukins" (per Hawking). En altres ocasions la imprecisió provoca un canvi total de significat; així, per exemple, s'escriu «Plutón" en lloc de "Platón". El nom d'Albert Einstein es mereix un comentari especific, ja que s'ha trobat escrit de setze formes distintes: "Aintan", "Aistan", "Anstain", “Anstein", «Eigsthen", «Ehinsteir», «Einsten”, «Eïsten», “Eister", «Hemstein", “Enisteny", “Enstaïn", "Haignsten", "Heintein", "Heinsteen" y "Heinstein".

Els equívocs entre un personatge i la seva aportació al coneixement científic aixi com els que poden derivar-se de paraules que sonen de forma semblant provoca situacions gracioses, com algunes de les que segueixen:
"Darwin inventà la teoria de l'ebullició" (6è. EGB) i "l'avaluació dels animals" (7è. EGB); Edison, "el motor de quatre temps" (8è. EGB)" i “la llum" (7è EGB); Einstein, "la penicil. lina ila dinamita" (5è EGB), "el material fotoelèctric, la radioactivitat i la bombeta atòmica" (6è. EGB), i "l'electricitat i el moviment" (7è. EGB); Galileo, "la catapulta" (8è. EGB); Gutenberg, "la màquina d'escriure i la taula periòdica dels minerals" (6è. EGB) i "l'empresa" (7è. EGB); Kepler, "la llei de la palanca" (8è. EGB); Newton, "el newton" (7è. EGB) i "el submari" (8è. EGB); Nobel, "els premis de literatura" (6è. EGB) i "la paraula" (8è. EGB); Pascal, "la vacuna antiràbica" (8è. EGB); Ramón y Cajal, "el sistema solar" (6è. EGB) i Volta, "els volts" (7è. EGB)".

Cal posar l'accent en la fantasia dels nens envers tots aquells artefactes, del món dels invents, que s'haurien de fabricar amb diferents propòsits. Així, n'hi ha de purament fantàstics: "una màquina del temps (6è. EGB)"; de tipus ecològic: "una màquina per a cosir els forats a la capa d'ozó (5è. EGB)"; d'utilitat: "Una alarma que tingui molts rajos que impedeixein els atracaments als bancs (6è. EGB) i de justícia social: "Més treball per a la gent i una màquina per a curar els malalts" (8è. EGB) i "una cosa perquè no existeixin els pobres (6è. EGB)".

\section{Conclusió general a tall de reflexió}

Sembla que els científics catalogats pels nens ho són més per una contribució de tipus social que no pas per la seva aportació científica o tecnològica, que molt sovint desconeixen.

També s'hauria d'aprofitar el coneixement que aquestes idees aporten, com a motivació per millorar la cultura científica dels alumnes.

\section{Agraïments}

Cal fer constar la col.laboració de Mercè Pàmies, Dolors Aymamí i Àngel Bernal, ex-alumnes de Ciències de l'Escola de Magisteri de Tarragona, així com la de la Sra. Montserrat Curto, professora de l'escola "Joan Miró" de Miami Platja, i la del professor Josep Lluís Recio, director del col.legi "Teresa Miquel" de Reus, i la dels col.legis "Montsant" $i$ “La Presentació", de Reus $i$ «Escola Annexa de Pràctiques", de Tarragona, perquè sense el seu valuós ajut aquest estudi no s'hauria dut a terme.

\section{Referències bibliogràfiques}

1 JOUD, D. La Generalitat crea una comisión para estimular la cultura científica. "La Vanguardia". 25 novembre 1989. Secció de "Ciencia y Tecnología". Pàg. 6.

${ }^{2}$ GILABERT, R.M. i GAVALDÄ, J. La cultura científica en alumnos de ingeniería química. "Actas de los XII Encuentros de didáctica de las Ciencias Experimentales". Oviedo. setembre 1991. 